SCIENTIFIC REPORT

\title{
Expression of pigment epithelium derived factor and vascular endothelial growth factor in choroidal neovascular membranes and polypoidal choroidal vasculopathy
}

\author{
M Matsuoka, N Ogata, T Otsuji, T Nishimura, K Takahashi, M Matsumura
}

Br J Ophthalmol 2004;88:809-815. doi: 10.1136/bjo.2003.032466

\begin{abstract}
Aims: To determine whether pigment epithelium derived factor (PEDF), a protein that inhibits angiogenesis, is expressed in human choroidal neovascular membranes (CNVMs) and in tissues from an eye with polypoidal choroidal vasculopathy (PCV). In addition, to compare the expression of PEDF with that of vascular endothelial growth factor (VEGF), a known stimulator of angiogenesis, in these tissues.

Methods: CNVMs, associated with age related macular degeneration (AMD), angioid streaks, and PCV, were obtained during surgery. The expression of PEDF and VEGF in the excised subretinal fibrovascular membranes was determined by immunohistochemistry.

Results: PEDF and VEGF were strongly expressed in the vascular endothelial cells and retinal pigment epithelial (RPE) cells in the CNVMs where numerous new vessels were prominent (clinically active CNVMs). On the other hand, immunoreactivity for PEDF and VEGF was weak in the new vessels where fibrosis was prominent (clinically quiescent CNVMs). However, the RPE cells were still positive for PEDF and VEGF. The specimens from the eye with PCV also showed strong expression of PEDF and VEGF in the vascular endothelial cells and the RPE cells.

Conclusion: Because PEDF is an inhibitor of ocular angiogenesis and an inhibitor of ocular cell proliferation, our results suggest that PEDF along with VEGF may modulate the formation of subfoveal fibrovascular membranes.
\end{abstract}

S ubfoveal fibrovascular membranes can lead to a loss of central vision in patients with age related macular degeneration (AMD), ocular histoplasmosis, high myopia, and other diseases. Most subfoveal fibrovascular membranes are caused by choroidal neovascularisations (CNVs), and the initial step in the formation of choroidal neovascular membranes (CNVMs) is the growth of new vessels from the choriocapillaris through Bruch's membrane into the subretinal space. ${ }^{1-4}$ Earlier studies of experimentally induced and surgically excised CNVMs have shown that vascular endothelial growth factor (VEGF), ${ }^{5-10}$ transforming growth factor $\beta$ $($ TGF- $\beta),{ }^{11}$ and basic fibroblast growth factor (bFGF) ${ }^{12-15}$ were expressed in the CNVMs, and these factors were considered to have a role in the development of the CNVMs.

Polypoidal choroidal vasculopathy (PCV) was first described by Yannuzzi et $a l^{16}{ }^{17}$ and is characterised by an abnormal vascular network of choroidal vessels with polyplike dilations at the terminals of the branches. Whether PCV represents abnormal vessels from the choroidal circulation or neovascularisation from choriocapillaris is still being debated. ${ }^{18-20}$ Relevant to this study, a recent investigation demonstrated that VEGF was expressed in the vascular endothelial cells and the retinal pigment epithelial (RPE) cells in PCV. ${ }^{2}$

Angiogenesis is controlled by a balance of factors that stimulate or inhibit new vessel formation. In normal tissues, the inhibitory influences predominate and neovascularisation does not occur..$^{22}$ In contrast, in a variety of pathological states, such as tumour growth, proliferative diabetic retinopathy, and choroidal neovascularisation, new vessels develop because of increased levels of angiogenic stimulators and/or decreased levels of inhibitors. ${ }^{24}$

Recent studies have demonstrated that pigment epithelium derived factor (PEDF) is a strong inhibitor of angiogenesis. ${ }^{25}{ }^{26}$ Thus, PEDF inhibited the migration of endothelial cells in vitro in a dose dependent manner and was more effective than angiostatin, thrombospondin-1, and endostatin. ${ }^{25}$ These results placed PEDF among the most potent natural inhibitor of angiogenesis.

VEGF is strongly expressed in human $\mathrm{CNVMs}^{5-8}$ and in specimens from $\mathrm{PCV}^{21}$ but the expression of PEDF in these subfoveal fibrovascular membranes has not been determined. Earlier, we demonstrated an upregulation of the mRNA and protein of PEDF in eyes with an experimentally induced choroidal neovascularisation and suggested that PEDF may modulate the development of CNVMs. ${ }^{27}$

Because of the counterbalancing effects of PEDF and VEGF, ${ }^{26}$ we hypothesised that PEDF will also be expressed in human CNVMs, and the levels of PEDF and VEGF will depend on the clinical status of the subfoveal fibrovascular membranes. To test this hypothesis, we have performed immunohistochemical studies on surgically excised subfoveal fibrovascular membranes and determined the level of expression of PEDF and VEGF.

\section{MATERIALS AND METHODS}

CNVMs, associated with AMD and angioid streaks, and tissues from an eye with PCV were obtained during surgery for the treatment of these membranes. The procedures conformed to the tenets of the Declaration of Helsinki, and a signed written consent was obtained from all patients. The excision of subfoveal fibrovascular membranes was performed on seven eyes of seven patients, and the surgical indications and procedures have been reported. ${ }^{28}$

\section{Preoperative clinical analysis}

The size of each subfoveal fibrovascular membrane was expressed as a percentage of the optic disc diameter after correlating it with the preoperative fluorescein angiograms. The subfoveal fibrovascular membranes were considered to

Abbreviations: $A M D$, age related macular degeneration; $C N V$, choroidal neovascularisation; PCV, polypoidal choroidal vasculopathy PEDF, pigment epithelium derived factor; VEGF, vascular endothelial growth factor 
Table 1 Patient data, clinical characteristics

\begin{tabular}{|c|c|c|c|c|c|c|}
\hline Case & Sex & Age & Diagnosis & Clinical phenomenon & Size & Active or quiescent \\
\hline 1 & $\mathrm{~F}$ & 58 & Angioid streaks & Fresh subretinal haemorrhage, serous retinal detachment & 1.0 & Active \\
\hline 2 & M & 86 & $A M D$ & PED, serous retinal detachment & 2.5 & Active \\
\hline 3 & M & 65 & AMD & Serous retinal detachment & 1.2 & Active \\
\hline 4 & $\mathrm{~F}$ & 82 & $A M D$ & Fresh subretinal haemorrhage, serous retinal detachment & 2.0 & Active \\
\hline 5 & $M$ & 70 & $\mathrm{AMD}$ & Subretinal haemorrhage, subretinal fibrosis & 0.8 & Quiescent \\
\hline 6 & $\mathrm{~F}$ & 72 & $A M D$ & Subretinal fibrovascular membrane & 2.5 & Quiescent \\
\hline 7 & M & 63 & PCV & Fresh subretinal haemorrhage, PED & 2.0 & Active \\
\hline
\end{tabular}

be at an active or quiescent stage by the clinical data, review of standard fundus photographs, optical coherence tomography (OCT), and fluorescein and indocyanine green (ICG) angiograms. A subfoveal fibrovascular membrane was considered to be active if fresh subretinal haemorrhages and serous retinal detachment were present, or hyperleakage was displayed in fluorescein and/or ICG angiograms. They were considered to be quiescent if severe gliosis or subretinal fibrosis was present, subretinal haemorrhages were not fresh, leakage was not marked, and only tissue staining was displayed in the angiograms.

\section{Tissue processing}

The surgically excised subfoveal fibrovascular membranes were fixed in $4 \%$ paraformaldehyde in phosphate buffered saline (PBS) at $4{ }^{\circ} \mathrm{C}$. The tissues were placed in $30 \%$ sucrose in PBS for 20 minutes at $4^{\circ} \mathrm{C}$, dehydrated by graded ethanol series, and then embedded in paraffin. Sections, $4 \mu \mathrm{m}$ thick, were deparaffinised and prepared for immunohistochemical study. Haematoxylin and eosin staining was also performed to determine the histological characteristics of the membranes.

\section{Immunohistochemical analysis for PEDF and VEGF}

To detect the proteins of PEDF or VEGF immunohistochemically, serial or very closely adjacent $4 \mu \mathrm{m}$ thick sections were used. Immunoperoxidase staining was carried out with the LSAB kit (Dako, Glostrup, Denmark) according to the manufacturer's protocol. All steps were performed at room temperature unless otherwise stated. Briefly, sections were deparaffinised, fixed in cold acetone $\left(4^{\circ} \mathrm{C}\right)$ for 10 minutes, and then treated with $3 \%$ hydrogen peroxide to block endogenous peroxidase activity. Then, affinity purified rabbit polyclonal primary antibody against human PEDF (BioProducts, Maryland, Inc, MD, USA, 1:300), or affinity purified rabbit polyclonal antibody against human VEGF (Cat No VEGF (A-20):sc-152, Santa Cruz Biotechnology, Inc, CA, USA, 1:200), was applied to sections for 60 minutes followed by incubation with biotinylated goat anti-rabbit IgG. The slides were then incubated with horseradish peroxidase (HRP) conjugated avidin. For a chromogen, 3-amino-9ethyl-carbazole (AEC) (Dako, Glostrup, Denmark), was used, and the slides were counterstained with methyl green. Between each step, the sections were washed three times with PBS. For control, preimmune rabbit IgG or mouse IgG was used instead of the primary antibody. Sections were observed under a light microscope.

The degree and pattern of immunostaining was assessed by masked observers. The intensity of labeling was graded: grade 0 , not stained; grade 1 , slightly stained; grade 2, moderately stained; grade 3, strongly stained.

\section{RESULTS}

\section{Preoperative clinical analysis}

The clinical characteristics of the patients are summarised in table 1. An active CNVM was characterised by subretinal haemorrhage and/or a serous retinal detachment, and hyperleakage from the CNVM by fluorescein angiography (fig l, case 1). A typical case of a quiescent CNVM showing subretinal fibrosis by ophthalmoscopy is shown in figure 2 (case 5). Fluorescein and ICG angiograms displayed only tissue staining and no leakage from the CNVM. A case of PCV showing subretinal haemorrhage and retinal pigment epithelium detachment (PED) by ophthalmoscopy is shown in figure 3. Fluorescein angiograms displayed fluorescein leakage from the lesion and fluorescein pooling corresponding to the PED. ICG angiograms revealed polyp-like lesions with an associated abnormal vascular network (case 7). These findings are characteristic of an active subfoveal fibrovascular membrane.

\section{Histopathological examination}

The surgically excised CNVMs were examined in haematoxylin and eosin sections. There were several variations in the make-up of the membranes that ranged from fibrotic membranes with a small number of vascular cells to membranes with diffuse and severe vascularisation. Neovascularisation was more prominent than fibrosis in cases 1 to 4 , and these were classified clinically as active CNVMs (fig 1D, E). Fibrosis and proliferation of RPE cells were more prominent than neovascularisation in cases 5 and 6, and these were classified as quiescent CNVMs clinically (fig 2D, E). This difference in the make-up of the membranes was not related to the type of disease-that is, AMD and angioid streaks, from which the membranes were obtained. All specimens contained RPE cells and other cell types, assumed to be fibroblasts and macrophages by their morphology, in the stroma in varying numbers.

The PCV case (case 7) had different histopathological characteristics from cases of CNVMs. The fibrovascular tissues that appeared to correspond to the polypoidal lesions were observed under the RPE cells and contained numerous dilated, thin walled vessels and massive fibrin-like materials (fig 3D, E, F).

\section{Immunoreactivity for PEDF and VEGF}

PEDF positive cells were detected to some degree in all specimens (table 2)-for example, RPE cells in the stroma, perivascular areas, and the RPE cells covering the CNVMs. The clinically active CNVMs showed strong immunoreactivity for PEDF in the RPE cells located in the perivascular areas and also in the endothelial cells of the numerous new vessels (table 2, cases 1, 2, 3, and 4, figs $1 F, G$ ). On the other hand, the clinically quiescent CNVMs showed weak immunoreactivity for PEDF in the new vessels where fibrosis was prominent but strong immunoreactivity for PEDF was detected in the RPE cells (table 2, cases 5 and 6; figs $2 \mathrm{~F}, \mathrm{G}$ ).

Immunoreactivity for VEGF was also detected in the RPE cells located in the perivascular areas and in the RPE cells covering the CNVMs. Especially strong reactivity was observed in the RPE cells located in the perivascular areas where numerous neovascular vessels were prominent in cases 

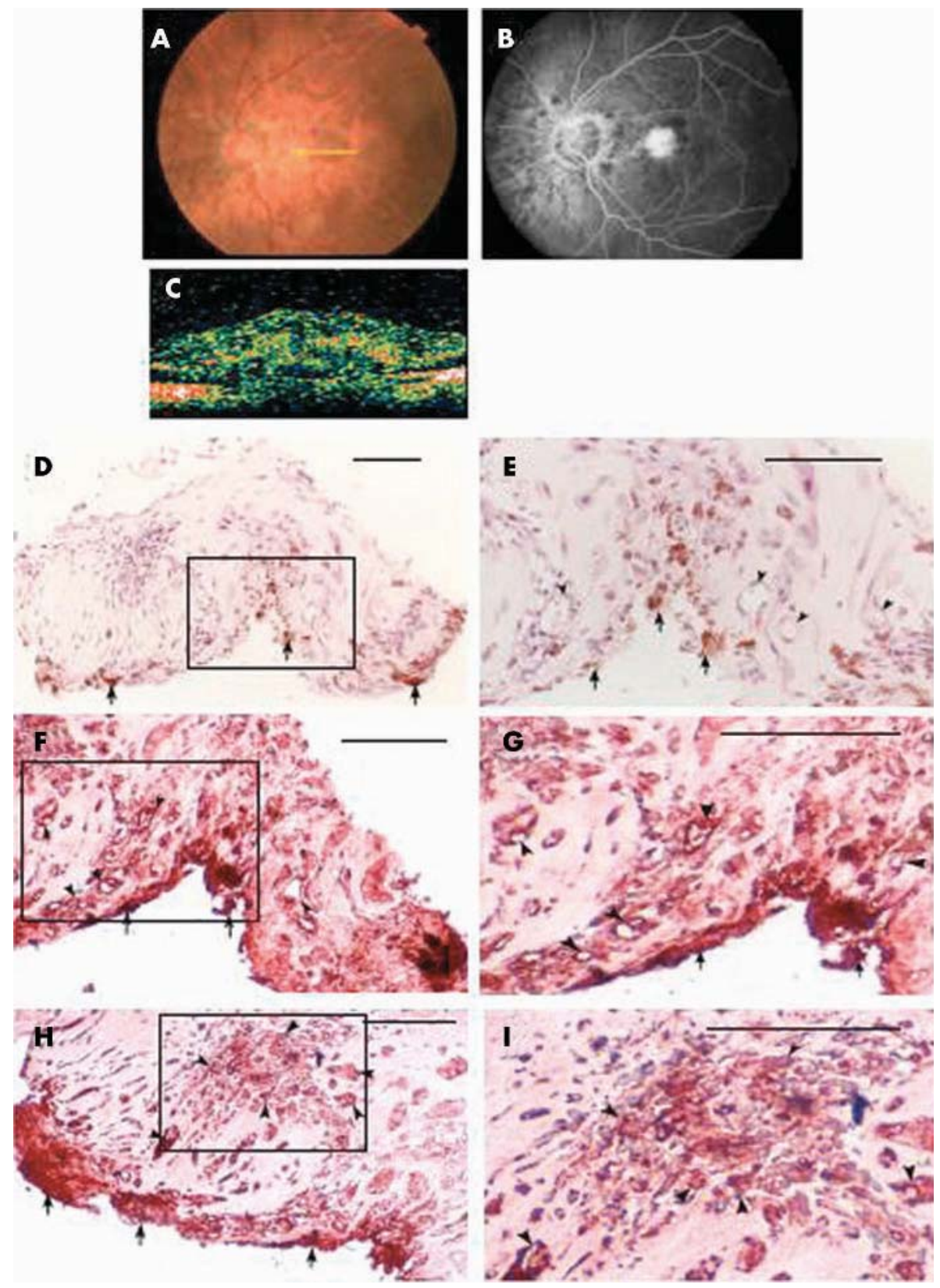

Figure 1 An active CNVM (case 1). (A) Preoperative fundus photograph. Subretinal haemorrhage and a serous retinal detachment can be seen. The yellow arrow points to the direction of the sectional OCT scan (C). (B) Preoperative fluorescein angiogram. Hyperfluorescein leakages from the CNVM can be seen. OCT of (A). (C) OCT shows an elevation of the sensory retina by a highly reflective mass above the original RPE layer. (D) Haematoxylin and eosin section showing prominent neovascular vessels. RPE cells (arrows) are observed on the choroidal side. (E) A high power photograph of the region outlined by the black box in (D). Numerous neovascular vessels (arrowheads) are present. (F) Immunohistochemistry for PEDF expression. Strong immunoreactivity for PEDF is observed in endothelial cells (arrowheads) within the neovascularisation and the RPE cells (arrows) located in the perivascular areas. (G) A high power photograph of the region outlined by the black box in (F). (H) Immunohistochemistry for VEGF expression. Strong immunoreactivity for VEGF is observed in endothelial cells (arrowheads) within the neovascularisation and the RPE cells (arrows). (I) A high power photograph of the region outlined by the black box in $(\mathrm{H})$. Scale bars, $50 \mu \mathrm{m}$.

1 to 4 . These cases showed active CNVMs clinically, and strong immunoreactivity for VEGF was also observed in endothelial cells of neovascularisation where numerous neovascular vessels were prominent (table 2, fig $1 \mathrm{H}, \mathrm{I}$ ).

In contrast, immunoreactivity for VEGF was weak or not detectable in the new vessels where fibrosis appeared to be predominant over neovascularisation, but was positive in the RPE cells located in the perivascular area and in the RPE cells covering the CNVMs (table 2, cases 5 and 6; figs $2 \mathrm{H}, \mathrm{I}$ ). These cases were classified as quiescent CNVMs clinically.

The specimens from the eye with PCV showed strong immunoreactivity for PEDF in the RPE cells located above the fibrovascular membrane and also in the endothelial cells of the numerous abnormal vessels (table 2, case 7; figs 3G, H). In addition, these cells also showed strong immunoreactivity for VEGF in the RPE cells located above the fibrovascular membrane and also in the endothelial cells of the numerous abnormal vessels (table 2, case 7; figs 3I, J).

These results showed that when subfoveal fibrovascular membranes are active, both VEGF and PEDF are trongly expressed in the endothelial cells, and when subfoveal fibrovascular membranes are quiescent, both VEGF and PEDF are barely expressed in the endothelial cells. 

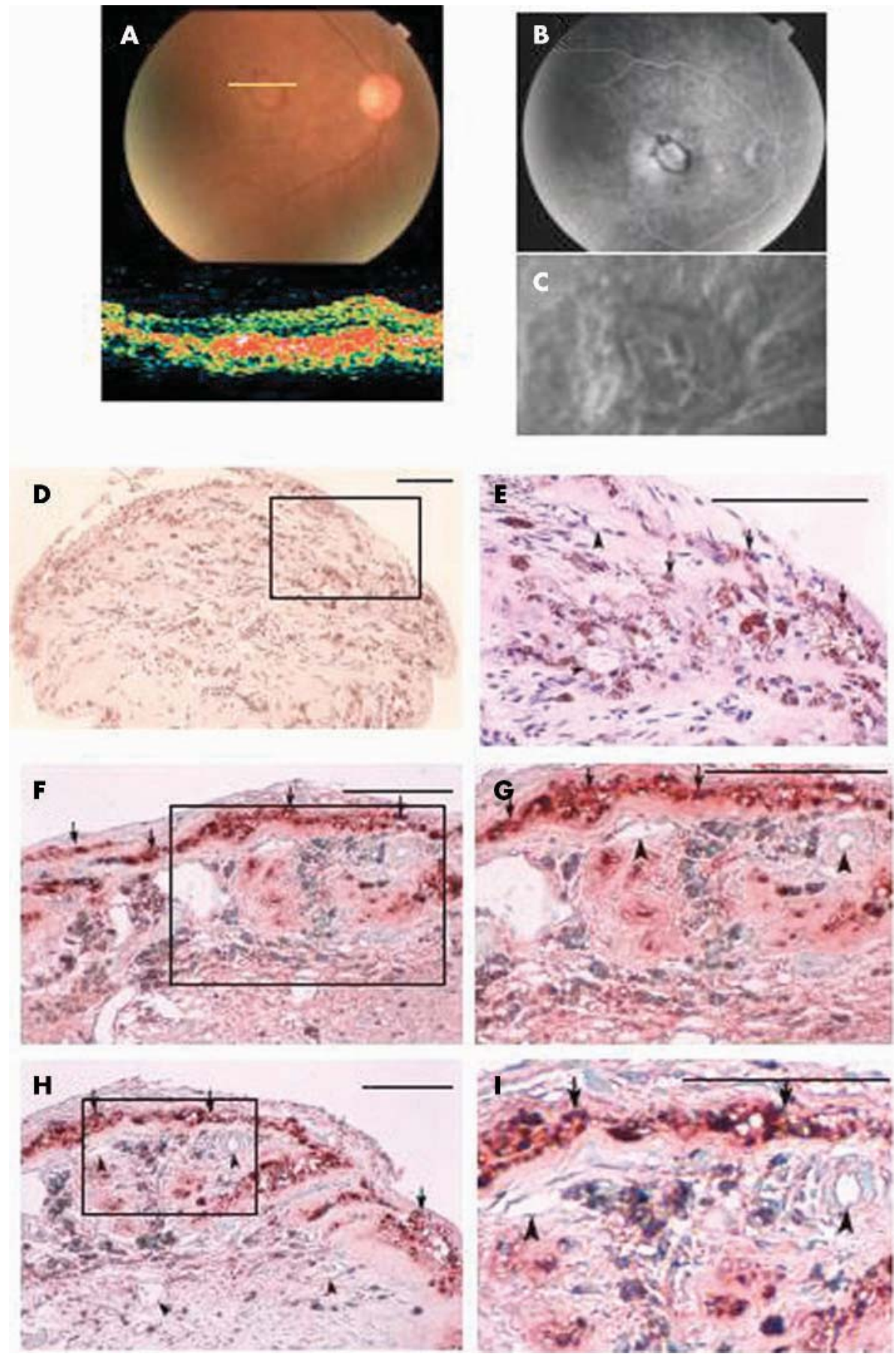

Figure 2 A quiescent CNVM (case 5). (A) Preoperative fundus photograph and OCT. Subretinal haemorrhage and fibrosis can be seen. The yellow arrow points to the direction of the sectional scan of OCT. OCT shows a thickening of a highly reflective layer corresponding to the RPE and choriocapillaris. High reflective layer consisting of red elements suggest fibrosis. (B) Preoperative fluorescein angiogram. No fluorescein leakage from the CNVM is observed. (C) Preoperative ICG angiogram. (D) ICG angiogram shows tissue staining in the CNVM with a dark rim. Haematoxylin and eosin section. (E) High power photograph of the region outlined by the black box in (D). Fibrosis and proliferation of RPE cells (arrows) are predominant over neovascular vessels (arrowheads). (F) Immunohistochemistry for PEDF expression. Strong immunoreactivity for PEDF is observed in the RPE cells (arrows) located in the perivascular area and the RPE cell layers covering the CNVMs. (G) A high power photograph of the region outlined by the black box in (F). Strong immunoreactivity for PEDF is observed in the RPE cells (arrows) but it is barely detectable in new vessels (arrowheads). (H) Immunohistochemistry for VEGF expression. Immunoreactivity for VEGF is observed in the RPE cells (arrows) located in the perivascular area and RPE cells covering the CNVM, whereas immunoreactivity for VEGF is weak or not detectable in the new vessels (arrowheads). (I) A high power photograph of the region outlined by the black box in $(\mathrm{H})$. Scale bars, $50 \mu \mathrm{m}$.

\section{DISCUSSION}

Angiogenesis is believed to develop from an imbalance between stimulators and inhibitors of angiogenesis. ${ }^{24}$ Thus, the PEDF level in the vitreous is decreased in eyes with proliferative diabetic retinopathy. ${ }^{29}{ }^{30}$ Gao et al also reported that the retinal PEDF levels, in contrast with that of VEGF, were negatively correlated with retinal neovascularisation. ${ }^{31}$ We, on the other hand, did not observe an upset in the 

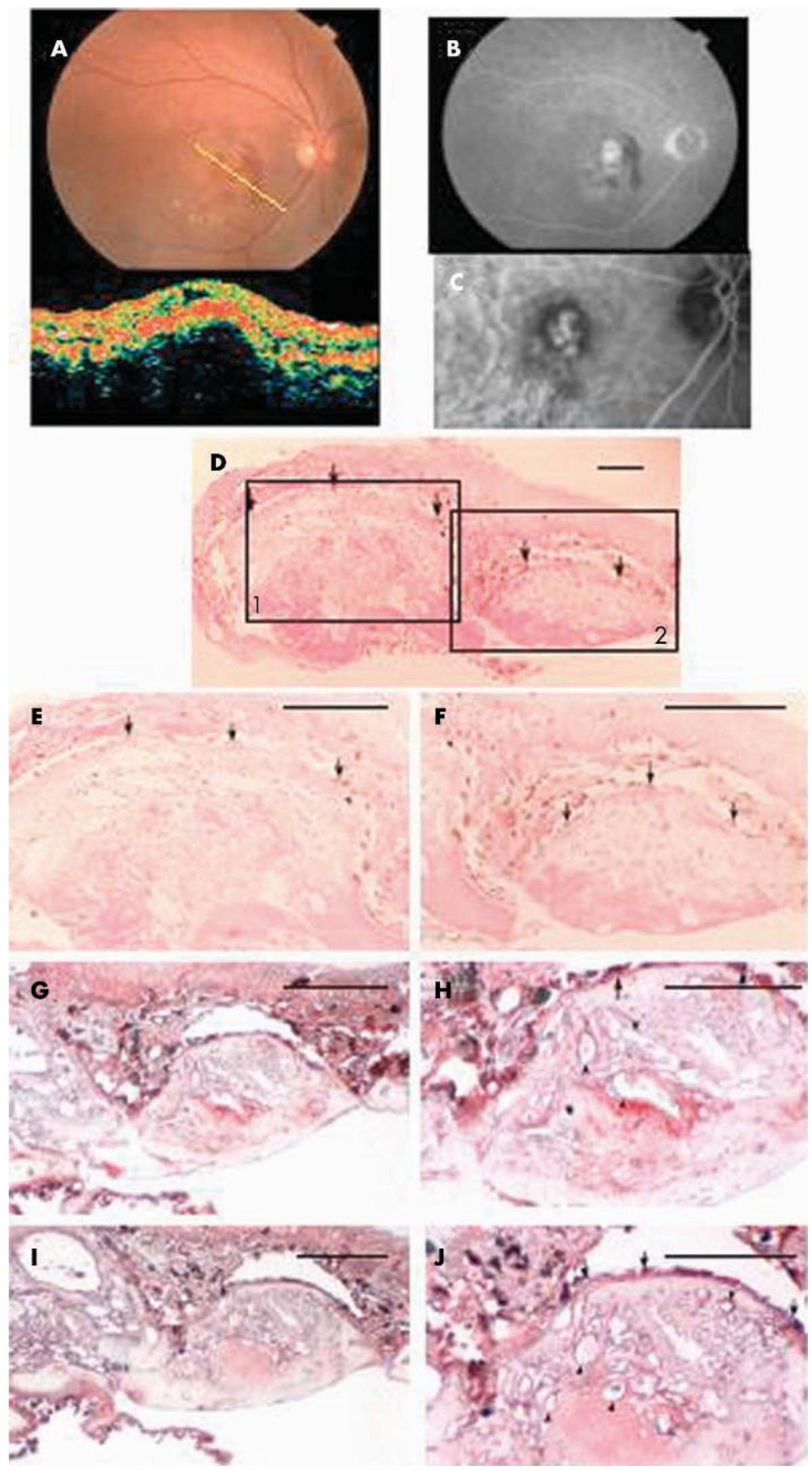

Figure 3 A case of PCV. (A) Preoperative fundus photograph and OCT. Subretinal haemorrhage and retinal pigment epithelium detachment (PED) are observed. The yellow arrow points to the direction of the sectional scan of OCT. OCT shows an elevation of the sensory retina by a highly reflective, dome-like layer. (B) Preoperative fluorescein angiogram displaying fluorescein leakage from the lesion and fluorescein pooling corresponding to the PED. (C) Preoperative ICG angiogram. (D) ICG angiogram displays polyp-like lesions with an associated abnormal vascular network. Haematoxylin and eosin section. The fibrovascular tissue that appears to correspond to the polypoidal lesions. The fibrovascular tissue is observed beneath the domelike elevation of the RPE layer (arrows). (E) A high power photograph of the region outlined by the black box 1 in (D). The fibrovascular tissue contains numerous dilated thin wall vessels and massive fibrin-like materials. (F) A high power photograph of the region outlined by the black box 2 in (D). The abnormal vessels are surrounded by fibrin-like materials. (G) Immunohistochemistry for PEDF expression. (H) A high power photograph of (G). Strong immunoreactivity for PEDF is observed in the RPE cells (arrows) located above the fibrovascular membrane and also detected in the endothelial cells of the numerous abnormal vessels (arrowheads). (I) Immunohistochemistry for VEGF expression. (J) A high power photograph of (I). Strong immunoreactivity for VEGF is observed in the RPE cells located above the fibrovascular membrane and also in the endothelial cells of the numerous abnormal vessels. Scale bars, $100 \mu \mathrm{m}$. 


\begin{tabular}{|c|c|c|c|c|}
\hline \multirow[b]{2}{*}{ Case } & \multicolumn{2}{|c|}{ PEDF immunoreactivity* } & \multicolumn{2}{|c|}{ VEGF immunoreactivity ${ }^{*}$} \\
\hline & RPE & Vascular endothelial cells & RPE & Vascular endothelial cells \\
\hline 1 & ++ & ++ & ++ & ++ \\
\hline 2 & ++ & + & ++ & ++ \\
\hline 3 & ++ & + & + & + \\
\hline 4 & ++ & + & + & ++ \\
\hline 5 & ++ & \pm & ++ & - \\
\hline 6 & ++ & $\overline{-}$ & + & - \\
\hline 7 & ++ & + & ++ & + \\
\hline
\end{tabular}

balance of VEGF and PEDF in the new vessels within the CNVMs in this study; the expression of VEGF was correlated with the expression of PEDF.

PEDF suppressed the VEGF induced proliferation and migration of vascular endothelial cells significantly. ${ }^{32}$ It was recently reported that plasminogen kringle 5 upregulated PEDF production in vascular endothelial cells of the retina. ${ }^{33}$ However, the regulation of PEDF gene expression and antiangiogenic mechanisms of PEDF is still unclear.

A recent study reported that the treatment of human carcinoma cells with hypoxia induced the expression of PEDF as well as VEGF. Moreover, treatment of these cells with VEGF enhanced the expression of PEDF mRNA and secretion of PEDF, and the addition of neutralising VEGF antibody substantially blocked PEDF expression. ${ }^{34}$ These data strongly indicate that VEGF upregulates PEDF production. Although the molecular mechanism behind the VEGF induced PEDF production is unknown, the authors suggested that VEGF enhances PEDF production, and PEDF regulates angiogenesis through a negative feedback and also regulates tumour cell growth.

A similar mechanism may be present in the pathological process of CNVM formation. We previously demonstrated the expression of PEDF and VEGF in experimentally induced CNV. ${ }^{27}$ When CNV was active, PEDF and VEGF were both strongly expressed in the choroidal neovascular lesions. Then after the CNV had developed, the expression of both VEGF and PEDF decreased in the endothelial cells, whereas strong expression of PEDF was present in the proliferated RPE cells covering the CNV. A previous study also demonstrated a significant increase in the vitreous levels of PEDF early after CNV induction in a rat model..$^{35}$ On the other hand, it has been reported that the vitreous has lower PEDF levels with no apparent change of VEGF levels in AMD patients with CNV. ${ }^{36}$ These findings indicate that the eyes of AMD patients with CNV have potential activity for angiogenesis. However, it is questionable whether the vitreous levels of PEDF and VEGF directly reflect the expression levels of these factors in CNVMs, because human CNV is usually only one local lesion in one eye and is quite small. Thus, we observed the expression of PEDF and VEGF within the tissues of subfoveal fibrovascular membranes.

PEDF may also inhibit the proliferation of ocular cells. ${ }^{37} \mathrm{It}$ is more likely that VEGF produced in endothelial cells enhances PEDF production, and PEDF regulates angiogenesis and cell proliferation through a negative feedback. This imbalance may contribute to the formation of CNVMs. Thus, clinically active subfoveal fibrovascular membranes were immunoreactive for PEDF and VEGF in the endothelial cells of new vessels. On the other hand, clinically quiescent subfoveal fibrovascular membranes were not immunoreactive for VEGF and PEDF in the endothelial cells. Neovascularisation at the quiescent stage would indicate that a competition between VEGF and PEDF was completed.

We have demonstrated that there was a decreased level of PEDF and an increased level of VEGF in the vitreous of eyes with diabetic retinopathy. ${ }^{30}$ In diabetic retinopathy and ischaemia induced retinal neovascularisation, the negative feedback mechanism by producing VEGF which regulates PEDF production, might be interrupted although the exact the mechanism is still unknown. Therefore, unbalanced expression of VEGF and PEDF that results in neovascularisation would occur.

It has been reported that differentiated human RPE cells express high levels of both VEGF and PEDF in vitro, and a critical balance between VEGF and PEDF is important to prevent the development of $\mathrm{CNV}^{38} \mathrm{~A}$ recent study also demonstrated that VEGF secreted by differentiated RPE cells upregulates PEDF expression via VEGFR-1 in an autocrine manner. ${ }^{39}$ These data suggest a regulatory interaction between the two counterbalancing systems; angiogenic stimulators and inhibitors are present in human RPE cells, and this balance might have a key role in maintaining the homeostasis of the human retina and also contributing to the formation of CNVs.

We found positive immunostaining for VEGF and PEDF in the RPE cells located in the stroma, perivascular areas, and in the layer covering CNVMs in all specimens. Several in vivo and in vitro studies have demonstrated that VEGF is produced constitutively by normal RPE cells, ${ }^{40-42}$ and VEGF expression has been reported in the RPE cells in CNV- ${ }^{8-10}$ Clinically, we have observed recurrent neovascularisation in what appeared to be quiescent CNVMs. Such recurrent neovascularisations usually occur with the disruption of the RPE layer covering the CNVMs. It is possible that such a recurrent neovascularisation may be induced by VEGF secreted from these RPE cells with a lack of VEGF induced PEDF gene upregulation for some reasons.

We found that the PEDF and VEGF expressions were strong in the new vessels in active CNVMs, whereas they were weak in the new vessels in quiescent CNVMs. These findings support our hypothesis that the levels of PEDF and VEGF depend on the clinical status of the subfoveal fibrovascular membranes. In this study, PEDF expression and VEGF expression in CNVM were parallel. These data suggest that in vivo VEGF induced PEDF gene upregulation and feedback mechanism may be present in CNVM.

In conclusion, the correlation of the expression of PEDF with the vascular status of the subfoveal fibrovascular membranes strongly suggests that PEDF along with VEGF plays an important part in choroidal neovascularisation and PCV.

\section{ACKNOWLEDGEMENTS}

This study was supported in part by a grant in aid for scientific research from the Ministry of Education in Japan and by the Japan Association for Inhibition of Blindness. 
Authors' affiliations

M Matsuoka, N Ogata, T Otsuji, T Nishimura, K Takahashi, M Matsumura, Department of Ophthalmology, Kansai Medical University, Moriguchi, Osaka 570-8507, Japan

Correspondence to: N Ogata, MD, PhD, Department of Ophthalmology, Kansai Medical University, Fumizono-cho 10-15, Moriguchi, Osaka 570-8507, Japan; ogata@takii.kmu.ac.jp

Accepted for publication 25 October 2003

\section{REFERENCES}

1 Green WR, Key SD. Senile macular degenetration: a histopathologic study. Trans Am Ophthalmol Soc 1977:75:180-254.

2 Green WR, McDonnell PJ, Yeo JH. Pathologic features of senile macular degeneration. Ophthalmology 1985;92:615-27.

3 D'Amore PA. Mechanisms of retinal and choroidal neovascularization. Invest Ophthalmol Vis Sci 1994;35:3974-79.

4 Heriot WJ, Henkind P, Bellhorn RW, et al. Choroidal neovascularization can digest Bruch's membrane: a prior break is not essential. Ophthalmology 1984;91:1603-8.

5 Kvanta A, Algvere PV, Berglin L, et al. Subfoveal fibrovascular membranes in age-related macular degeneration express vascular endothelial growth factor. Invest Ophthalmol Vis Sci 1996;37:1929-34.

6 Reddy VM, Zamore RL, Kaplan HJ. Distribution of growth factors in subfoveal fibrovascular membranes in age-related macular degeneration and presumed ocular histoplasmosis syndrome. Am J Ophthalmol 1995;120:291-301.

7 Amin R, Puklin JE, Frank RN. Growth factor localization in choroidal neovascular membranes of age-related macular degeneration. Invest Ophthalmol Vis Sci 1994;35:3178-88.

8 Lopez PF, Sippy BD, Lamber HM, et al. Transdifferentiated retinal pigment epithelial cells are immunoreactive for vascular endothelial growth factor in surgically excised age-related macular degeneration-related choroidal neovascular menbranes. Invest Ophthalmol Vis Sci 1996;37:855-68.

9 Yi X, Ogata N, Komada M, et al. Vascular endothelial growth factor expression in choroidal neovascularization in rats. Graefes Arch Clin Exp Ophthalmol 1997:235:313-19.

10 Wada M, Ogata N, Otsuji T, et al. Expression of vascular endothelial growth factor and its receptor (KDR/flk-1) mRNA in experimental choroidal neovascularization. Curr Eye Res 1999;18:201-13.

11 Ogata N, Yamamoto C, Miyashiro M, et al. Expression of transforming growth factor- $\beta$ mRNA in experimental choroidal neovascularizarion. Curr Eye Res 1997; 16:9-18.

12 Amin RH, Frank RN, Eliot D, et al. Vascular endothelial growth factor (VEGF) and basic fibroblast growth factor (bFGF) immunoreactivity in human choroidal neovascular membranes. Invest Opthalmol Vis Sci 1995;36:S2565

13 Ogata N, Matsushima M, Takada Y, et al. Expression of basic fibroblast growth factor mRNA in developing choroidal neovascularization. Curr Eye Res 1996;15:1008-18.

14 Matsushima M, Ogata N, Takada Y, et al. Expression of fibroblast growth factor receptor 1 in experimental choroidal neovascularization with in situ hybridization. Jpn J Ophthalmol 1996:40:329-38.

15 Frank RN, Amin RH, Eliott D, et al. Basic fibroblast growth factor and vascular endothelial growth factor are present in epiretinal and choroidal neovascula membranes. Am J Ophthalmol 1996;122:393-403.

16 Yannuzzi LA. Idiopathic popypoidal choroidal vasculopathy. Presented at the 1982 Macula Society meeting..

17 Yannuzzi LA, Sorenson J, Spaide RF, et al. Idiopathic popypoidal choroidal vasculopathy (IPCV). Retina 1990;10:1-8.

18 Yannuzzi LA, Wong DW, Sforzolini BS, et al. Popypoidal choroidal vasculopathy and neovascularized age-related macular degenetration. Arch Ophthalmol 1999;117:1503-10.

19 Uyama M, Matsubara T, Fukuchima I, et al. Idiopathic polypoidal choroidal vasculopathy in Japanese patients. Arch Ophthalmol 1999;117:1035-42.
20 Okubo A, Sameshima M, Uemura A, et al. Clinicopathological correlation of polypoidal choroidal vasculopathy revealed by ultrastructual study. Br J Ophthalmol 2002;86:1093-8.

21 Terasaki H, Miyake $Y$, Nakamura $M$, et al. Popypoidal choroidal vasculopathy treated with macular translocation: clinical pathological correlation. Br J Ophthalmol 2002;86:321-7.

22 Bouck N. Tumor angiogenesis: the role of oncogenes and tumor suppressor genes. Cancer Cells 1990;2:179-85.

23 Hanahan D, Folkman Y. Patterns and emerging mechanisms of angiogenic switch during tunorigenesis. Cell 1996;86:353-64.

24 Folkman J. Clinical applications of research on angiogenesis. N Engl J Med 1995;333:1757-63.

25 Dawson DW, Volpert OV, Gillis $\mathrm{P}$, et al. Pigment epithelium-derived factor: a potent inhibitor of angiogenesis. Science 1999;285:245-8.

26 Stellmach V, Crawford SE, Zhou W, et al. Prevention of ischemia-induced retinopathy by the natural ocular antiangiogenic agent pigment epitheliumderived factor. Proc Natl Acad Soc USA 2001;98:2593-7.

27 Ogata N, Wada M, Otsuji T, et al. Expression of pigment epithelium-derived factor in normal adult rat eye and experimental choroidal neovascularization. Invest Ophthalmol Vis Sci 2002;43:1 168-75.

28 Lambert HM, Capone A Jr, Aaberg TM, et al. Surgical excision of subfoveal neovascular membranes in age-related macular degeneration. Am J Ophthalmol 1992; 113:257-62.

29 Ogata N, Tombran-Tink J, Nishikawa M, et al. Pigment epithelium-derived factor in the vitreous is low in diabetic retinopathy and high in rhegmatogenous retinal detachment. Am J Ophthalmol 2001;132:378-82.

30 Ogata N, Nishikawa M, Nishimura T, et al. Unbalanced vitreous levels of pigment epithelium-derived factor and vascular endothelial growth factor in diabetic retinopathy. Am J Ophthalmol 2002;134:348-53.

31 Gao G, Li Y, Zhang D, et al. Unbalanced expression of VEGF and PEDF in ischemia-induced retinal neovascularizarion. FEBS Lett 2001;489:270-6.

32 Duh EJ, Yang HS, Suzuma I, et al. Pigment epithelium-derived factor suppresses ischemia-induced retinal neovascularization and VEGF-induced migration and growth. Invest Ophthalmol Vis Sci 2002;43:821-9.

33 Gao G, Li Y, Gee S, et al. Down-regulation of VEGF and up-regulation of PEDF: a possible mechanism for the anti-angiogenic activity of plasminogen kringle 5. J Biol Chem 2002;277:9492-7.

34 Miyagishi D, Ohno MK, Amagasa T, et al. Regulation of the expression of pigment epithelium-derived factor in human oral squamous cell cartinoma cell line. Cancer Lett 2003;196:77-85.

35 Renno RZ, Youssri Al, Michaud N, et al. Expression of pigment epitheliumderived factor in experimental choroidal neovascularization. Invest Ophthalmol Vis Sci 2002;43:1574-80.

36 Holekamp NM, Bouck N, Volpert O. Pigment epithelium-derived factor is deficient in the vitreous of patients with choroidal neovascularization due to age-related macular degeneration (1). Am J Ophthalmol 2002;134:220-7.

37 Ogata N, Nishikawa M, Nishimura T, et al. Inverse levels of pigment epithelium-derived factor and vascular endothelial growth factor in the vitreous of eyes with rhegmatogenous retinal detachment and proliferative vitreoretinopathy. Am J Ophthalmol 2002;133:851-2.

38 Ohono-Matsui K, Morita I, Tombran-Tink J, et al. Novel mechanism for agerelated macular degeneration: an equilibrium shift between the angiogenesis factors VEGF and PEDF. J Cell Physiol 2001;189:323-33.

39 Ohono-Matsui K, Yoshida T, et al. Vascular endothelial growth factor upregulates pigment epithelium-derived factor expression via VEGFR-1 in human retinal pigment epithelial cells. Biochem Biophy Res Comm 2003;303:962-7

40 Adamis AP, Shima DT, Yeo KT, et al. Synthesis and secretion of vascular permeability factor/vascular endothelial growth factor by human retinal pigment epithelial cells. Biochem Biophys Res Comm 1993;193:631-8.

41 Yi X, Mai LC, Uyama M, et al. Time-course expression of vascular endothelial growth factor as related to the development of the retinochoroidal vasculature in rats. Exp Brain Res 1998;193:155-60.

42 Ogata N, Yamanaka R, Yamamoto C, et al. Expression of vascular endothelial growth factor and its receptor, KDR, following retinal ischemia-reperfusion injury in the rat. Curr Eye Res 1998;17:1087-96. 